\title{
UPLC-MS/MS Determination of Linezolid and Heme in Plasma of Infected Patients and Correlation Analysis
}

\author{
Yingying Wang, ${ }^{1}$ Xuemei Ye, ${ }^{1}$ Qin Lan, ${ }^{1}$ Xiaofang Ke, ${ }^{1}$ Lufeng Hu $\mathbb{D}^{1},{ }^{1}$ and Lichuan Hu $\mathbb{D}^{2}$ \\ ${ }^{1}$ Department of Pharmacy, The First Affiliated Hospital of Wenzhou Medical University, Wenzhou 325000, China \\ ${ }^{2}$ Department of Pharmacy, Wenzhou Central Hospital, Wenzhou 325000, China
}

Correspondence should be addressed to Lufeng Hu; hulufeng79@sina.com and Lichuan Hu; hulipipi@126.com

Received 21 January 2021; Revised 8 June 2021; Accepted 18 June 2021; Published 12 July 2021

Academic Editor: Ali Imran

Copyright ( 2021 Yingying Wang et al. This is an open access article distributed under the Creative Commons Attribution License, which permits unrestricted use, distribution, and reproduction in any medium, provided the original work is properly cited.

\begin{abstract}
Linezolid can cause serious haematological toxicity, such as thrombocytopenia and aneamia. Heme, composed of iron and porphyrin, is an important component of hemoglobin. In order to investigate the relationship between the concentration of linezolid and heme in the plasma of infected patients, a UPLC-MS/MS method that can determine the concentrations of linezolid and heme simultaneously was developed and validated. A total of 96 healthy subjects and 81 infected patients, who received blood routine blood tests, were included and determined by the UPLC-MS/MS method. The results showed that the concentration of linezolid was $5.08 \pm 3.46 \mu \mathrm{g} / \mathrm{mL}$ in infected patients who were treated with linezolid. The heme in healthy subjects was $7.05 \pm 8.68 \mu \mathrm{g} / \mathrm{mL}$, and it was significantly decreased to $0.88 \pm 0.79 \mu \mathrm{g} / \mathrm{mL}$ in infected patients $(P<0.01)$. Spearman correlation analysis showed that linezolid had a high negative correlation with platelet (PLT) $(R=-0.309)$. Heme had a high positive correlation with hemoglobin $(\mathrm{Hb})(R=0.249)$ in healthy subjects and infected patients. The ROC analysis showed that heme had diagnostic value to distinguish low $\mathrm{Hb}(110 \mathrm{~g} / \mathrm{L})$. In conclusion, there was a positive correlation between heme and $\mathrm{Hb}$, and this correlation was also observed in infected patients. A high concentration of linezolid was inclined to decrease PLT. Monitoring of heme and linezolid helps in the early diagnose of low $\mathrm{Hb}$ and PLT.
\end{abstract}

\section{Background}

Linezolid, the first new class of oxazolidinones antibiotics [1], is used for the treatment of multidrug-resistant grampositive pathogens, including vancomycin-resistant enterococci (VRE), methicillin-resistant staphylococcus aureus (MRSA), and several anaerobes [2]. Moreover, linezolid has been recommended for the treatment of patients with multidrug-resistant (MDR) or extensively drug-resistant (XDR) tuberculosis [3]. However, linezolid causes high incidences of severe side effects, such as haematological toxicity, hyperlactatemia, metabolic acidosis, gastrointestinal disturbances, and peripheral neuropathy [4]. The most common is haematological toxicity, with the incidence of thrombocytopenia and anemia at $32 \%-45.8 \%$ and $25 \%$, respectively [5]. Hanai et al. reported that thrombocytopenia and anemia developed in $48.4 \%$ and $10.4 \%$ of patients during linezolid therapy [6].
Heme is an essential substance, which can be synthesized by bacteria and human cells [7]. It is composed of iron and porphyrin and considered as an important component of many proteins, such as oxidase protein $[8,9]$, cytochrome protein [10], hemoglobin, and cytochrome P450 protein [11]. Moreover, heme is essential for electron transport [12], metabolism of oxygen molecules and diatomic gases [13], and various redox reactions [14], and thus, plays important roles in a number of metabolic pathways of organisms.

Some studies have been carried out to investigate the toxic mechanisms of linezolid, such as mitochondrial ribosome inhibition [15], tissue-dependent mitotoxicity [4], deficiency of mitochondria encoded proteins [16], and similarities between human and bacterial ribosomes [17]. Those studies revealed part of the reason for adverse hematologic and peripheral nervous side effects, however, few of them focused on the heme, and the exact mechanism of its haematological toxicity is still unclear. Considering the important physiological function of 
heme, this study is aimed at developing a UPLC-MS/MS method to determine the heme and linezolid levels in infected patients and to investigate the correlation between them.

\section{Methods}

2.1. Ethics Statement and Subjects. This study was approved by the Ethics Committee of the First Affiliated Hospital of Wenzhou Medical University (2020-zz-219) and conducted in accordance with the Declaration of Helsinki. Data from all infected patients and healthy subjects were analyzed anonymously and securely. All data are for the use of investigators only.

2.2. Reagents and Instruments. Heme and linezolid were purchased from Sigma-Aldrich (Lewis, USA, lot: J0804A) and TRC (Toronto, Canada, lot: L466500). Fluconazole (purity > 98\%, lot: 481850) was purchased from national institutes for food and drug control (Peking, China). Acetonitrile, methanol, and formic acid were purchased from Merck Company (Darmstadt, Germany), and all reagents were at the HPLC grade. Ultrapure water (resistance $>18 \mathrm{~m} \Omega$ ) was prepared by a Millipore Milli-Q purification system (Bedford, USA).

The ACQUITY UPLC system consists of a Binary Solvent Manager (BSM) and a Sample Manager with a FlowThrough Needle (SM-FTN). Heme and linezolid were analyzed using the Xevo TQ-S Micro triple quadrupole mass spectrometer (Waters Corporation, USA) equipped with an electrospray ion source (ESI).

2.3. UPLC-MS/MS Determination of Linezolid and Heme. Chromatographic separation of linezolid, heme, and fluconazole (IS) was conducted at BEH C18 column $(2.1 \mathrm{~mm} \times$ $100 \mathrm{~mm}, 1.7 \mu \mathrm{m}$ Waters Corporation) at $40^{\circ} \mathrm{C}$. The mobile phase consists of $0.1 \%$ formic acid water (A), and acetonitrile (B) was used in gradient elution as follows: (Tmin/acetonitrile): $0.0-0.5 / 20 \%, \quad 0.5-0.8 / 80 \%, 0.8-2.5 / 80 \%$, and $2.5-$ $3.0 / 20 \%$. The flow rate was set at $0.3 \mathrm{~mL} / \mathrm{min}$.

Linezolid, heme, and IS were detected by multiple reaction monitoring (MRM) mode. The UPLC-MS/MS conditions are listed in Table 1. The desolvation temperature was $600^{\circ} \mathrm{C}$, cone gas flow was $150 \mathrm{~L} / \mathrm{hr}$, and desolvation gas flow was $1000 \mathrm{~L} / \mathrm{hr}$. The injection volume was $0.5 \mu \mathrm{L}$. All UPLCMS/MS data were collected and processed by Masslynx 4.1 software (Waters Corp, MA, USA).

2.4. Calibration Curve and Sample Preparation. The stock solution of heme was prepared in alkaline solution at a concentration of $1.00 \mathrm{mg} / \mathrm{mL}$ ( $1 \mathrm{~mL}$ water added with $5 \mu \mathrm{L}$ saturated sodium hydroxide), and linezolid was prepared in methanol at $1.00 \mathrm{mg} / \mathrm{mL}$. The calibration standards were prepared by spiking $5 \mu \mathrm{L}$ mixed standard solutions of linezolid and heme into $45 \mu \mathrm{L}$ plasmas. The added concentrations of standard curve samples were $0.5,1,2,4,8,16$, and $32 \mu \mathrm{g} / \mathrm{mL}$.

The $50 \mu \mathrm{L}$ plasma samples were precipitated by $200 \mu \mathrm{L}$ of $1 \%$ formic acid-acetonitrile, supplemented with $0.05 \mu \mathrm{g} / \mathrm{mL}$ of IS. Then, the mixture was vortexed for $0.2 \mathrm{~min}$, centrifuged at $15000 \mathrm{rpm}$ for $5 \mathrm{~min}$, and $0.5 \mu \mathrm{L}$ supernatant was injected into the UPLC-MS/MS system for analysis.
TABLE 1: UPLC-MS/MS conditions of linezolid, heme, and (IS).

\begin{tabular}{lccccc}
\hline Compound & $\begin{array}{c}\text { Ion } \\
\text { mode }\end{array}$ & $\begin{array}{c}\text { Parent ion } \\
(\mathrm{m} / \mathrm{z})\end{array}$ & $\begin{array}{c}\text { Daughter } \\
\text { ion }(\mathrm{m} / \mathrm{z})\end{array}$ & $\begin{array}{c}\text { Cone } \\
(\mathrm{V})\end{array}$ & Collision \\
\hline Linezolid & Positive & 338.03 & 296.02 & 10 & 15 \\
Heme & Positive & 616.0 & 557.0 & 10 & 35 \\
IS & Positive & 307.1 & 238.1 & 6 & 16 \\
\hline
\end{tabular}

2.5. Method Validation. Precision, precision, recovery, matrix effect, and stability of the method were verified with 2,4 , and $8 \mu \mathrm{g} / \mathrm{mL}$ quality control samples. Diurnal precision of heme and linezolid was assessed at three quality control levels, repeated three times a day, and for three consecutive days.

The extraction recovery was evaluated by comparing the peak area of heme in pure standard solution at the same concentration. The matrix effect was investigated by comparing the peak area of heme with the same concentration in the extracted samples under three quality control levels. The stability of the three QC samples was tested at $2 \mathrm{~h}, 4 \mathrm{~h}$, and $24 \mathrm{~h}$ at room temperature.

2.6. Infected Patients and Healthy Subjects. The subjects involved in this study were infected patients and healthy subjects from the First Affiliated Hospital of Wenzhou Medical University. All patients underwent regular clinical biochemical examinations, including blood routine test (BRT) and liver and kidney function examination. After completing the routine blood test, the blood samples of healthy subjects will be collected and stored at $-80^{\circ} \mathrm{C}$ for heme detection. Blood samples were collected for the determination of linezolid and heme in infected persons receiving linezolid treatment.

The BRT and biochemical indices were analyzed with Beckman AU5800 biochemical measurement and Sysmex XE-2100 automated hematology analyzer. Linezolid and heme were determined by the developed UPLC-MS/MS method.

2.7. Statistical Analysis. The differences of BRT between infected patients and healthy subjects were analyzed by using independent samples test. The relationship between linezolid and heme and BRT was analyzed by Spearman's bivariate correlation. The receiver operating characteristic curve (ROC) was used to evaluate the diagnostic value of linezolid and heme. All statistical differences were analyzed using SPSS software 17 .

\section{Results}

3.1. UPLC-MS/MS Determination of Heme and Linezolid. According to the optimized UPLC and mass conditions, the typical mass spectrums and UPLC-MS/MS chromatograms are shown in Figure 1. Heme, linezolid, and IS were eluted at $1.74 \mathrm{~min}, 1.63 \mathrm{~min}$, and $1.59 \mathrm{~min}$, respectively. No endogenous compounds interfere with heme, linezolid, and IS in positive ion mode.

The calibration curve of heme and linezolid showed a good linear relationship in the concentration range of $0.5-32 \mu \mathrm{g} / \mathrm{mL}$. 


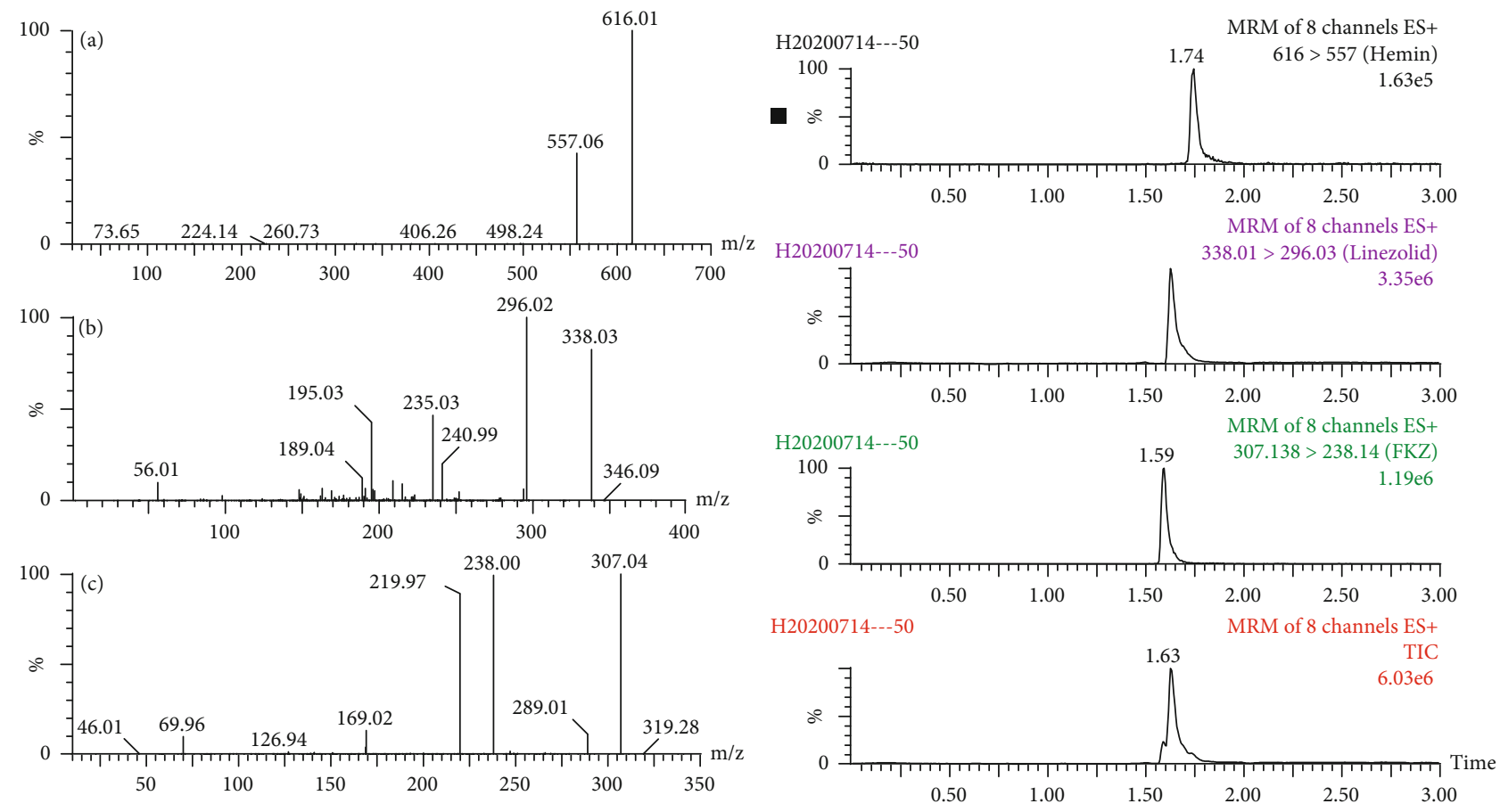

FIGURE 1: UPLC-MS/MS mass spectrogram and chromatogram of heme (a), linezolid (b), and IS (c).

TABLE 2: The precision, extraction recovery, and matrix effects of linezolid and heme.

\begin{tabular}{|c|c|c|c|c|c|}
\hline \multirow{2}{*}{ Compound } & \multirow{2}{*}{$\mu \mathrm{g} / \mathrm{mL}$} & \multicolumn{2}{|c|}{ Precision (RSD) } & \multirow{2}{*}{ Extraction recovery (mean $\pm \mathrm{SD}, \%)$} & \multirow{2}{*}{ Matrix effects (mean $\pm \mathrm{SD}, \%$ ) } \\
\hline & & Intraday & Interday & & \\
\hline \multirow{4}{*}{ Linezolid } & 3 & 9.10 & 11.22 & $86.45 \pm 12.47$ & $83.99 \pm 11.24$ \\
\hline & 6 & 8.20 & 14.53 & $85.12 \pm 11.95$ & $77.02 \pm 6.78$ \\
\hline & 12 & 2.19 & 12.53 & $75.37 \pm 7.64$ & $75.31 \pm 7.01$ \\
\hline & 3 & 4.32 & 5.01 & $77.46 \pm 1.86$ & $98.76 \pm 12.06$ \\
\hline \multirow[t]{2}{*}{ Heme } & 6 & 3.67 & 3.31 & $78.83 \pm 6.40$ & $95.40 \pm 9.61$ \\
\hline & 12 & 1.72 & 3.76 & $77.30 \pm 8.47$ & $83.54 \pm 6.48$ \\
\hline
\end{tabular}

The regression equation and coefficient of heme are as follows: $y=0.1727 x-0.1132, R^{2}=0.9953$; and those of linezolid are as follows: $y=15.938 x-1.1024, R^{2}=0.9983$. According to the signal-to-noise ratio $(\mathrm{S} / \mathrm{N})$ of 3 , the detection limit of heme was $1.3 \mathrm{ng} / \mathrm{mL}$, and that of Linezolid was $0.5 \mathrm{ng} / \mathrm{mL}$.

The relative standard deviation (RSD) of intraday and interday precision of heme and linezolid was less than $15 \%$, the extraction recovery was over $75 \%$, and the matrix effect was over $83 \%$. The results are shown in Table 2 . The stability RSD of both heme and linezolid at room temperature was no more than $15 \%$.

3.2. Clinical Indexes of Infected Patients and Subjects. A total of 81 infected patients (64 males, 17 females) and 96 healthy subjects ( 35 males, 61 females) were included, with average ages of $61.99 \pm 17.21$ and $43.89 \pm 11.48$ years old, respectively. Linezolid concentrations, BRT, and renal and liver function tests of a total of 107 infected patients were recorded. The differences in BRT and renal and liver function tests between healthy subjects and infected patients are listed in Tables 3 and 4 . The results showed that the WBC, percentage of neutrophil, and the absolute value of neutrophil and monocytes in infected patients were higher than those of healthy subjects $(P<0.05)$, while the RBC, Hb, and HCT in infected patients were lower $(P<0.05)$. Renal and liver function tests showed that indirect bilirubin, total protein, and albumin decreased, while direct bilirubin, ALT, AST, ALP, $\gamma$-GT, and BUN increased in infected patients.

3.3. Heme Level in Healthy Subjects and Infected Patient. Based on the developed UPLC-MS/MS method, the mean concentration of heme in healthy subjects was $7.05 \pm 8.68 \mu$ $\mathrm{g} / \mathrm{mL}$, while the mean heme concentration in infected patients was significantly decreased to $0.88 \pm 0.79 \mu \mathrm{g} / \mathrm{mL}$ $(P<0.01)$. Spearman correlation analysis showed that heme had a high positive correlation with $\mathrm{RBC}(R=0.290), \mathrm{Hb}$ $(R=0.249)$, and HCT $(R=0.333)$ in healthy subjects. In the infected patients, heme was highly positively correlated with $\mathrm{Hb}$ and $\operatorname{HCT}(R=0.214, P=0.027)$. The correlation analysis between heme BRT indexes is shown in Figure 2 and 
TABLE 3: BRT indices of healthy subjects and infected patients (mean \pm SD).

\begin{tabular}{|c|c|c|c|}
\hline Indices & Healthy & Patient & $P$ \\
\hline White blood cell (WBC) & $5.88 \pm 1.30$ & $9.85 \pm 5.99$ & $<0.001$ \\
\hline Percentage of neutrophil & $0.55 \pm 0.07$ & $0.74 \pm 0.14$ & $<0.001$ \\
\hline Percentage of eosinophils & $0.02 \pm 0.01$ & $0.02 \pm 0.04$ & 0.672 \\
\hline Percentage of basophils & $0.0015 \pm 0.0018$ & $0.0013 \pm 0.0018$ & 0.504 \\
\hline Percentage of monocytes & $0.07 \pm 0.06$ & $0.07 \pm 0.04$ & 0.52 \\
\hline Percentage of lymphocytes & $0.36 \pm 0.08$ & $0.17 \pm 0.11$ & $<0.001$ \\
\hline Absolute value of eosinophils & $0.15 \pm 0.26$ & $0.19 \pm 0.39$ & 0.468 \\
\hline Absolute value of neutrophil & $3.24 \pm 0.99$ & $7.82 \pm 5.75$ & $<0.001$ \\
\hline Absolute value of monocytes & $0.40 \pm 0.13$ & $0.61 \pm 0.39$ & $<0.001$ \\
\hline Absolute value of lymphocyte & $2.08 \pm 0.53$ & $1.32 \pm 0.60$ & $<0.001$ \\
\hline Absolute value of basophils & $0.01 \pm 0.02$ & $0.01 \pm 0.02$ & 0.182 \\
\hline Red blood cell (RBC) & $4.64 \pm 0.38$ & $3.19 \pm 0.63$ & $<0.001$ \\
\hline Hemoglobin $(\mathrm{Hb})$ & $141.57 \pm 12.10$ & $94.40 \pm 19.56$ & $<0.001$ \\
\hline Hematocrit (HCT) & $0.41 \pm 0.05$ & $0.28 \pm 0.05$ & $<0.001$ \\
\hline Mean corpuscular volume & $89.55 \pm 3.02$ & $89.11 \pm 7.70$ & 0.598 \\
\hline Mean hemoglobin & $30.65 \pm 1.40$ & $29.67 \pm 2.45$ & 0.001 \\
\hline Mean hemoglobin concentration & $334.76 \pm 44.89$ & $333.42 \pm 15.97$ & 0.773 \\
\hline RBC volume distribution width & $12.83 \pm 0.63$ & $15.07 \pm 2.48$ & $<0.001$ \\
\hline $\mathrm{SD}$ value of $\mathrm{RBC}$ volume distribution & $84.21 \pm 414.70$ & $48.56 \pm 9.40$ & 0.375 \\
\hline Platelet (PLT) & $237.65 \pm 47.91$ & $237.06 \pm 128.52$ & 0.966 \\
\hline Thrombocytocrit & $0.26 \pm 0.05$ & $0.25 \pm 0.12$ & 0.800 \\
\hline Mean platelet volume & $10.89 \pm 0.85$ & $10.70 \pm 1.30$ & 0.226 \\
\hline SD value of platelet distribution & $13.18 \pm 1.88$ & $13.16 \pm 3.36$ & 0.950 \\
\hline Large platelet ratio & $32.13 \pm 7.18$ & $30.31 \pm 10.26$ & 0.154 \\
\hline
\end{tabular}

TABLE 4: hepatic and renal function indices of healthy subjects and infected patients (mean \pm SD).

\begin{tabular}{lccc}
\hline Indices & Healthy & Patient & $P$ \\
\hline Total bilirubin & $12.20 \pm 3.48$ & $19.66 \pm 37.72$ & 0.057 \\
Direct bilirubin & $3.98 \pm 1.10$ & $13.43 \pm 34.67$ & 0.009 \\
Indirect bilirubin & $8.32 \pm 2.61$ & $6.20 \pm 4.23$ & $<0.001$ \\
Total protein & $74.92 \pm 3.87$ & $62.82 \pm 8.72$ & $<0.001$ \\
Albumin & $45.21 \pm 2.52$ & $32.16 \pm 6.01$ & $<0.001$ \\
Globulin & $29.73 \pm 3.50$ & $30.66 \pm 7.11$ & 0.269 \\
Alanine transaminase (ALT) & $17.03 \pm 7.86$ & $41.39 \pm 57.53$ & $<0.001$ \\
Aspartate transaminase (AST) & $20.97 \pm 4.35$ & $73.79 \pm 244.11$ & 0.035 \\
Alkaline phosphatase (ALP) & $71.28 \pm 20.25$ & $141.82 \pm 111.15$ & $<1.40 \pm 69.26$ \\
$\gamma$-Glutamyltransferase ( $\gamma$-GT) & $21.17 \pm 10.00$ & $9.77 \pm 9.31$ & $<0.001$ \\
Blood urea nitrogen (BUN) & $4.94 \pm 1.20$ & $137.97 \pm 174.90$ & $<0.001$ \\
Creatinine & $65.63 \pm 12.51$ & $303.15 \pm 167.53$ & $<0.001$ \\
Uric acid & $302.91 \pm 61.10$ & & $<0.001$ \\
\hline
\end{tabular}




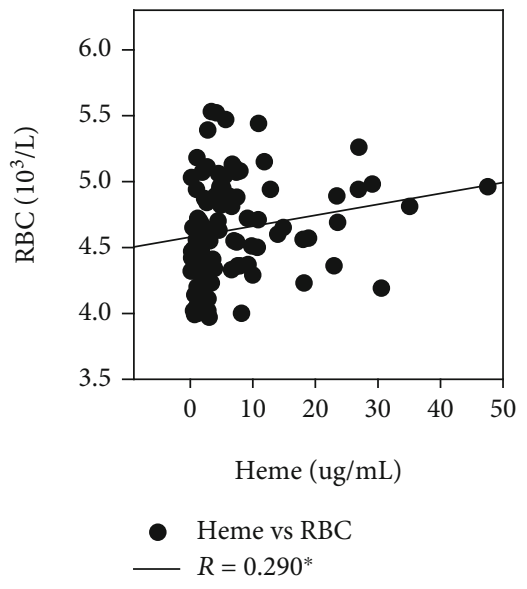

(a)

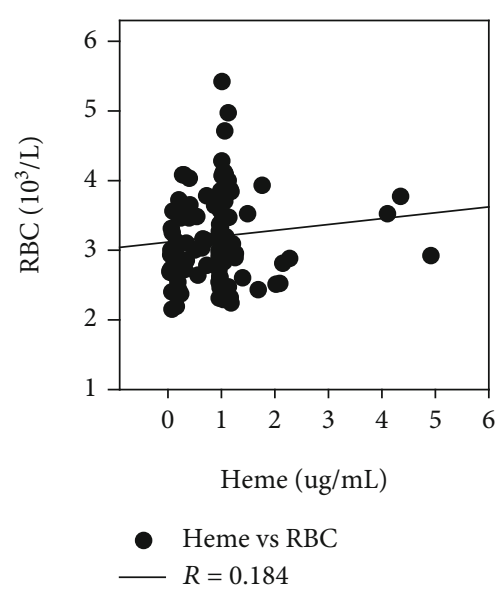

(d)

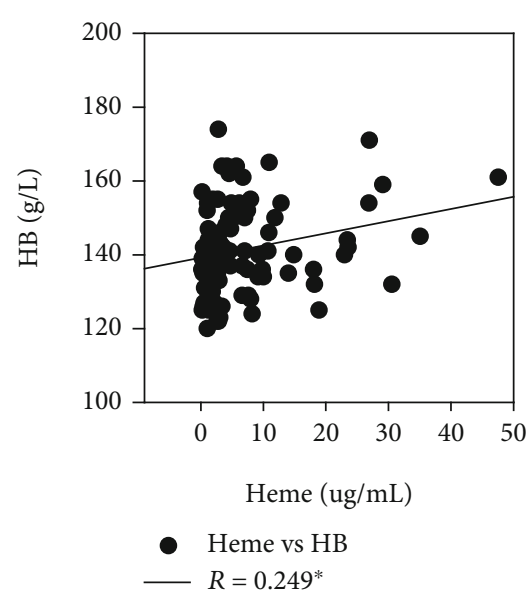

(b)

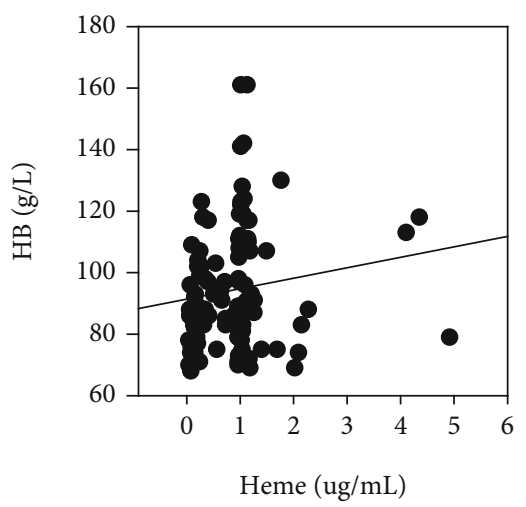

- Heme vs HB $R=0.214^{*}$

(e)

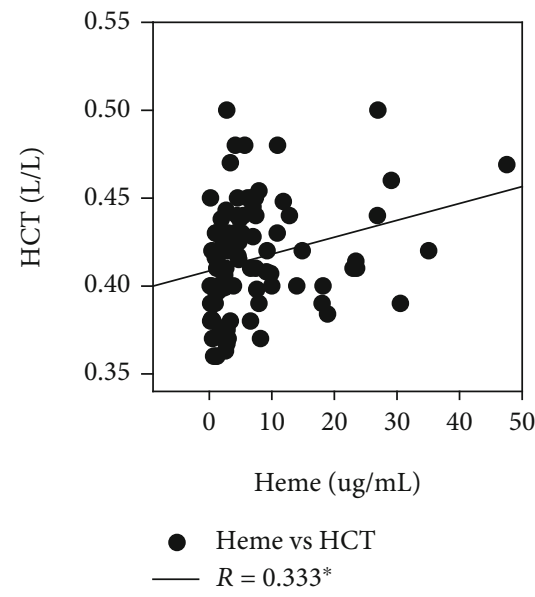

(c)

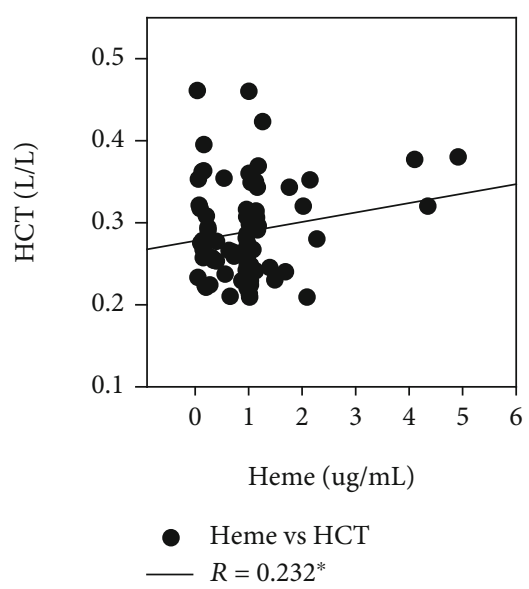

(f)

FIGURE 2: Correlation of heme with RBC, Hb, and HCT in healthy subjects (ABC) and infected patients (DEF). R: correlation coefficient of Spearman's analysis. $* P<0.05$.

Supplementary Table 1. Further ROC analysis (Figure 3) showed that heme was more valuable than linezolid in the diagnosis of low HB $(110 \mathrm{~g} / \mathrm{L})$.

3.4. Correlation Analysis of Linezolid and Heme in Infected Patients. Based on the developed UPLC-MS/MS method, we found that the mean serum concentration of linezolid was $5.08 \pm 3.46 \mu \mathrm{g} / \mathrm{mL}$. Spearman correlation analysis showed that linezolid was negatively correlated with WBC, neutrophil, monocytes, and PLT $(R=-0309)$ and positively correlated with eosinophils and lymphocytes. However, there was no correlation between RBC, $\mathrm{HB}$, and heme. The correlations between linezolid and RBC, Hb, PLT, heme, and other indices are shown in Figure 4 and Supplementary Table 2.

\section{Discussion}

So far, it has been reported that spectral deconvolution [18] and high-performance liquid chromatography diode array spectrophotometry [19] have been used to determine heme. In terms of HPLC-MS/MS, Fyrestam and Ostman [20]

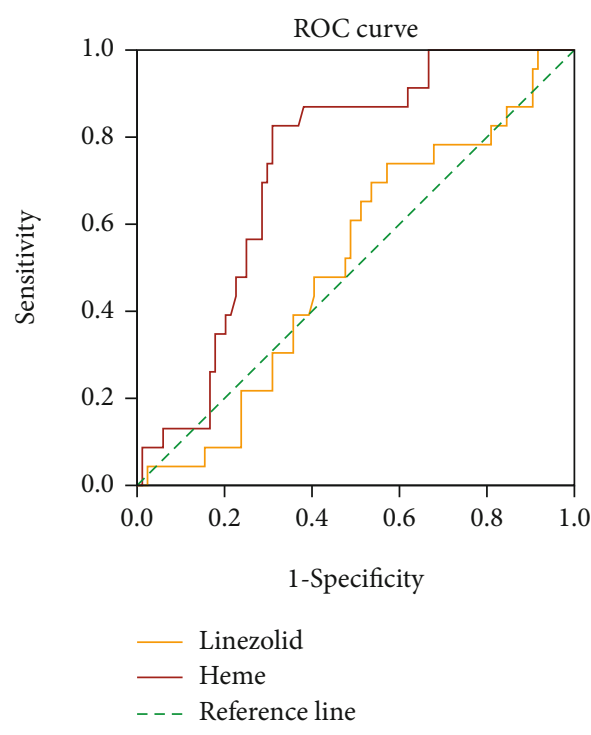

FIGURe 3: The ROC analysis of heme (0.731) and linezolid (0.515) in infected patients. 


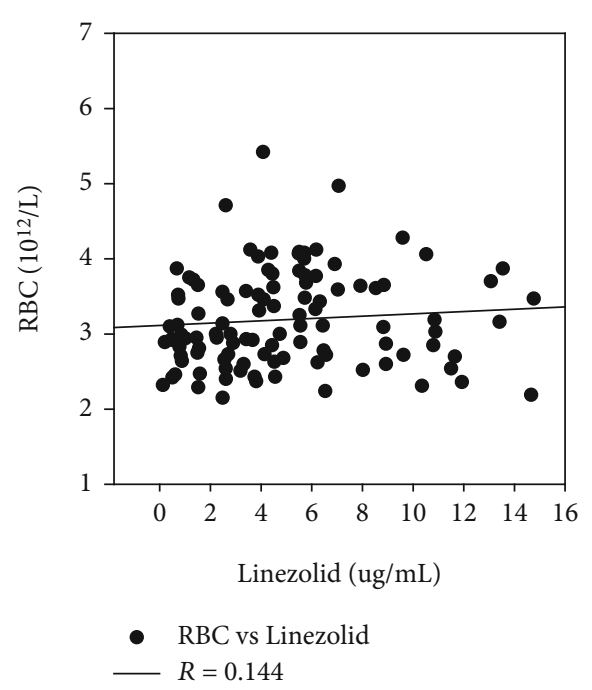

(a)

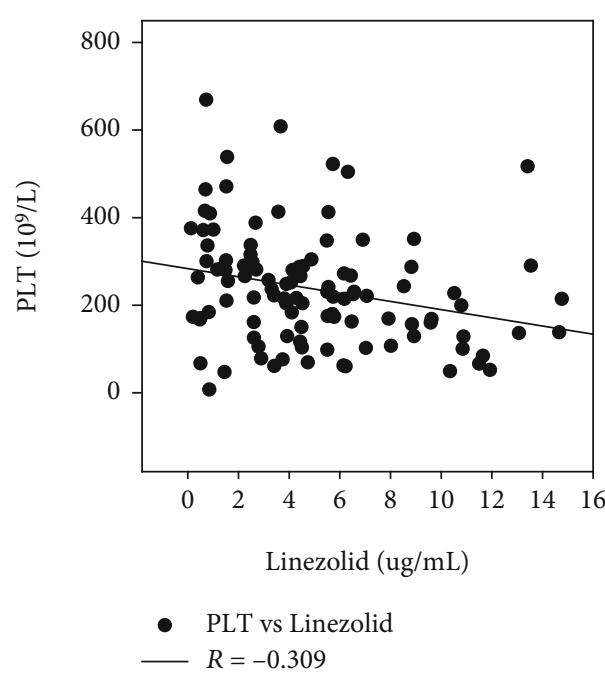

(c)

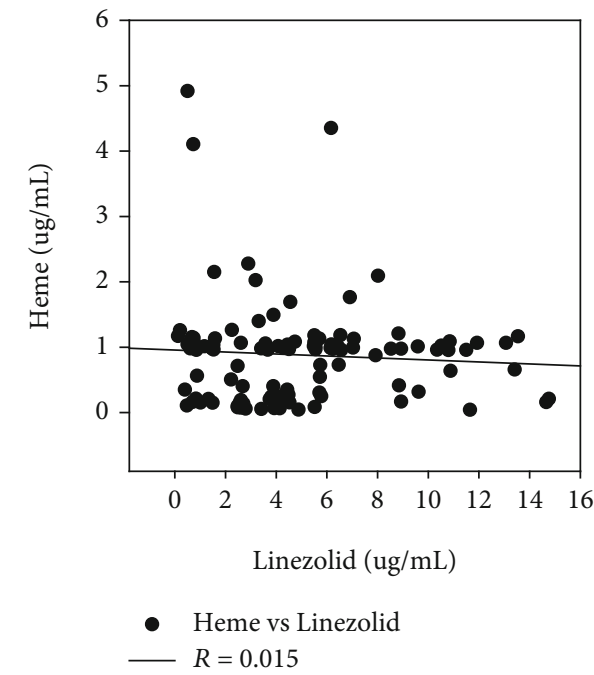

(b)

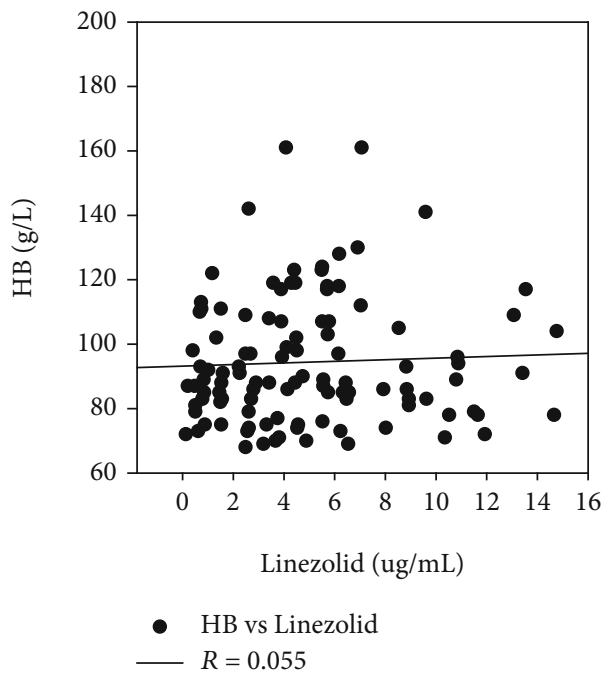

(d)

Figure 4: Correlation of linezolid with RBC, Hb, PLT, and heme in infected patients (DEF). R: correlation coefficient of Spearman's analysis. $* P<0.05$.

developed a liquid-liquid extraction method for the determination of heme in microorganisms by HPLC-MS/MS. Whiteaker et al. developed matrix-assisted laser desorption/ionization time-of-flight mass spectrometry (MALDITOFMS) for the determination of heme (ferriprotoporphyrin IX) in bacillus spores [21]. However, these methods are rarely used for the determination of heme in plasma.

Linezolid can be determined by HPLC-UV [22], UHPLC-PDA [23], quinone-based fluorophores [24], and LC-MS-MS [25]. HPLC-UV and LC-MS-MS are two widely used methods in clinical practice. Compared with HPLC, LC-MS-MS is simple and sensitive and specific. Moreover, LC-MS-MS can simultaneously determine multiple substances. So far, although several methods for the determination of linezolid in plasma have been reported, most of them are about simultaneous determination with other drugs, such as meropenem and theophyllin [26], piperacillin and teicoplanin [27], and daptomycin and tedizolid [28].
In this study, an UPLC-MS/MS method for simultaneous determination of heme and linezolid in plasma was developed for the first time. The results of method validation showed that our method was fast, convenient, and precise, and can be used to determine the heme and linezolid levels in patients.

In this study, 81 infected patients were included in this study. The blood, sputum, and urine culture tests showed that the main infectious bacteria were staphylococcus aureus, escherichia coli, and acinetobacter baumannii. The BRT, renal, and liver function tests showed that WBC, percentage of neutrophil, direct bilirubin, ALT, AST, ALP, and BUN increased. It indicated that there were obvious infected states in those infected patients.

So far, most studies have investigated the heme oxygenase- 1 in patients with various infections $[29,30]$, such as enterohemorrhagic Escherichia coli [31] and Mannheimia haemolytica infection [32], but few studies have focused on 
heme. In order to evaluate the heme level in infected patients, we included healthy subjects and infected patients. The Spearman correlation analysis showed that heme was related to WBC (0.221), RBC (0.290), and HB (0.249) in healthy subjects and had statistics difference $(P<0.05)$. While, in infected patients, only HB was related to heme $(0.214, P<$ $0.05)$. It is indicated that there is a stable connection between heme and $\mathrm{Hb}$, which does not change with infection.

It has been widely acknowledged that there is a significant correlation between the plasma concentration of linezolid and hemotoxicity in infected patients [33]. Even in healthy volunteers, linezolid intravenously reduced $\mathrm{Hb}$ and RBC levels [34]. Therefore, it is necessary to determine the plasma concentration of linezolid in infected patients. Our results showed that the plasma concentration of linezolid, which ranged from 0.5 to $14.7 \mu \mathrm{g} / \mathrm{mL}$, was highly correlated with PLT, but not with $\mathrm{Hb}, \mathrm{RBC}$, and heme. Dou et al. reported when $\mathrm{AUC}_{24}>243 \mathrm{mg} \cdot \mathrm{h} / \mathrm{L}$ or $C_{\min } \geq 6.3 \mathrm{mg} / \mathrm{L}$, the probability of thrombocytopenia was $>50 \%$ [35]. These results indicated that PLT was more sensitive to the serum concentration of linezolid than $\mathrm{RBC}$ and $\mathrm{HB}$. In other words, the higher the concentration of linezolid, the lower the PLT level.

\section{Conclusions}

A sensitive, reliable, and accurate UPLC-MS/MS for simultaneous determination of heme and linezolid was developed. The positive correlation between heme and $\mathrm{Hb}$ was stable and did not change with different infections. Compared with healthy subjects, the heme level was significantly decreased in infected patients $(P<0.01)$. Linezolid decreased PLT but not $\mathrm{RBC}$ and HB. Monitoring heme and linezolid can help in the early diagnose of low $\mathrm{Hb}$ and PLT.

\section{Data Availability}

The datasets generated and/or analyzed during the current study are not publicly available but are available from the corresponding author on reasonable request.

\section{Conflicts of Interest}

The authors declare no conflict of interest.

\section{Authors' Contributions}

Yingying Wang contributed equally to this work.

\section{Acknowledgments}

This work was supported by the fund of the Wenzhou Science and Technology Bureau (Y20180106, Y2020830) and the Health Department of Zhejiang Province (2021KY781).

\section{Supplementary Materials}

Supplementary material included Supplementary Table 1, Table 2, and ethical approval documents. (Supplementary Materials)

\section{References}

[1] D. L. Stevens, B. Dotter, and K. Madaras-Kelly, "A review of linezolid: the first oxazolidinone antibiotic," Expert Review of Anti-Infective Therapy, vol. 2, no. 1, pp. 51-59, 2004.

[2] Y. C. Chuang, J. T. Wang, H. Y. Lin, and S. C. Chang, "Daptomycin versus linezolid for treatment of vancomycin-resistant enterococcal bacteremia: systematic review and meta-analysis," BMC Infectious Diseases, vol. 14, no. 1, p. 687, 2014.

[3] A. A. Agyeman and R. Ofori-Asenso, "Efficacy and safety profile of linezolid in the treatment of multidrug-resistant (MDR) and extensively drug-resistant (XDR) tuberculosis: a systematic review and meta-analysis," Annals of Clinical Microbiology and Antimicrobials, vol. 15, no. 1, p. 41, 2016.

[4] G. Garrabou, A. Soriano, T. Pinós et al., "Influence of mitochondrial genetics on the mitochondrial toxicity of linezolid in blood cells and skin nerve fibers," Antimicrob Agents Chemother, vol. 61, no. 9, 2017.

[5] L. Moraza, L. Leache, I. Aquerreta, and A. Ortega, "Linezolidinduced haematological toxicity," Farmacia Hospitalaria, vol. 39, 6 pages, 2015.

[6] Y. Hanai, K. Matsuo, M. Ogawa et al., "A retrospective study of the risk factors for linezolid-induced thrombocytopenia and anemia," Journal of Infection and Chemotherapy, vol. 22, no. 8, pp. 536-542, 2016.

[7] N. Frankenberg, J. Moser, and D. Jahn, "Bacterial heme biosynthesis and its biotechnological application," Applied Microbiology and Biotechnology, vol. 63, no. 2, pp. 115-127, 2003.

[8] A. Jayaram, "525: autophagy induction and expression of the $70 \mathrm{kDa}$ heat shock protein and heme oxidase- 1 in peripheral blood mononuclear cells in human gestation," American Journal of Obstetrics and Gynecology, vol. 214, no. 1, 2016.

[9] N. Wijayanti, T. Kietzmann, and S. Immenschuh, "Heme oxygenase- 1 gene activation by the $\mathrm{NAD}(\mathrm{P}) \mathrm{H}$ oxidase inhibitor 4-(2-aminoethyl) benzenesulfonyl fluoride via a protein kinase B, p38-dependent signaling pathway in monocytes," Journal of Biological Chemistry, vol. 280, no. 23, pp. 21820 21829, 2005.

[10] M. Breuer, K. M. Rosso, and J. Blumberger, "Electron transport in the bacterial deca-heme cytochrome MtrF," Abstracts of Papers of the American Chemical Society, vol. 246, 2013.

[11] J. Y. Zhuang, A. R. Reddi, Z. H. Wang, B. Khodaverdian, E. L. Hegg, and B. R. Gibney, "Evaluating the roles of the heme a side chains in cytochrome c oxidase using designed heme proteins," Biochemistry, vol. 45, no. 41, pp. 12530-12538, 2006.

[12] K. Módis, V. M. S. Ramanujam, A. A. Govar et al., "Cystathionine- $\gamma$-lyase (CSE) deficiency increases erythropoiesis and promotes mitochondrial electron transport via the upregulation of coproporphyrinogen III oxidase and consequent stimulation of heme biosynthesis," Biochemical Pharmacology, vol. 169, 2019.

[13] S. H. Chang, J. Garcia, M. S. Kilberg, and A. Agarwal, "Induction of heme oxygenase- 1 by glucose deprivation is mediated by reactive oxygen species (center dot ROS) via the mitochondrial electron transport chain," Journal of the American Society of Nephrology, vol. 13, pp. 289a-290a, 2002.

[14] K. Mobius, R. Arias-Cartin, D. Breckau et al., "Heme biosynthesis is coupled to electron transport chains for energy generation," Proceedings of the National Academy of Sciences of the United States of America, vol. 107, no. 23, pp. 10436-10441, 2010. 
[15] O. K. Abou Hassan, M. Karnib, R. El-Khoury, G. Nemer, M. Ahdab-Barmada, and P. BouKhalil, "Linezolid toxicity and mitochondrial susceptibility: a novel neurological complication in a Lebanese patient," Frontiers in Pharmacology, vol. 7, 2016.

[16] A. S. de Vriese, R. van Coster, J. Smet et al., "Linezolid-induced inhibition of mitochondrial protein synthesis," Clinical Infectious Diseases, vol. 42, no. 8, pp. 1111-1117, 2006.

[17] R. Mishra, H. Patel, B. Goel, and T. Vakde, "A case of linezolid toxicity presenting as a sepsis mimic," Case Reports in Critical Care, vol. 2019, Article ID 2157674, 4 pages, 2019.

[18] J. Hamm, J. Y. Oh, J. D. Lebensburger, and R. P. Patel, "Simultaneous determination of free heme and free hemoglobin in biological samples by spectral deconvolution," Blood, vol. 124, no. 21, 2014.

[19] J. Wakamatsu, H. Odagiri, T. Nishimura, and A. Hattori, "Quantitative determination of Zn protoporphyrin IX, heme and protoporphyrin IX in Parma ham by HPLC," Meat Science, vol. 82, no. 1, pp. 139-142, 2009.

[20] J. Fyrestam and C. Ostman, "Determination of heme in microorganisms using HPLC-MS/MS and cobalt(III) protoporphyrin IX inhibition of heme acquisition in Escherichia coli," Analytical and Bioanalytical Chemistry, vol. 409, no. 30, pp. 6999-7010, 2017.

[21] J. R. Whiteaker, C. C. Fenselau, D. Fetterolf, D. Steele, and D. Wilson, "Quantitative determination of heme for forensic characterization of Bacillus spores using matrix-assisted laser desorption/ionization time-of-flight mass spectrometry," Analytical Chemistry, vol. 76, no. 10, pp. 2836-2841, 2004.

[22] S. Fortuna, G. De Pascale, E. Ragazzoni, M. Antonelli, and P. Navarra, "Validation of a new HPLC-UV method for determination of the antibiotic linezolid in human plasma and in bronchoalveolar lavage," Biomedical Chromatography, vol. 27, no. 11, pp. 1489-1496, 2013.

[23] V. Ferrone, M. Carlucci, R. Cotellese et al., "Development and validation of a fast micro-extraction by packed sorbent UHPLC- PDA method for the simultaneous determination of linezolid and ciprofloxacin in human plasma from patients with hospital-acquired pneumonia," Talanta, vol. 164, pp. 6468, 2017.

[24] B. A. Moussa, M. A. Mahrouse, M. A. Hassan, and M. G. Fawzy, "Spectrofluorimetric determination of gemifloxacin mesylate and linezolid in pharmaceutical formulations: application of quinone-based fluorophores and enhanced native fluorescence," Acta Pharmaceutica, vol. 64, no. 1, pp. 15-28, 2014.

[25] O. A. Phillips, M. E. Abdel-Hamid, and N. A. Al-Hassawi, "Determination of linezolid in human plasma by LC-MSMS," Analyst, vol. 126, no. 5, pp. 609-614, 2001.

[26] A. K. Attia, M. A. Al-Ghobashy, G. M. El-Sayed, and S. M. Kamal, "Simultaneous determination of linezolid, meropenem and theophylline in plasma," Data Brief, vol. 21, pp. 150-153, 2018.

[27] D. Ferrari, M. Ripa, S. Premaschi, G. Banfi, A. Castagna, and M. Locatelli, "LC-MS/MS method for simultaneous determination of linezolid, meropenem, piperacillin and teicoplanin in human plasma samples," Journal of Pharmaceutical and Biomedical Analysis, vol. 169, pp. 11-18, 2019.

[28] R. Tanaka, M. Kai, K. Goto et al., "High-throughput and widerange simultaneous determination of linezolid, daptomycin and tedizolid in human plasma using ultra-performance liquid chromatography coupled to tandem mass spectrometry," Journal of Pharmaceutical and Biomedical Analysis, vol. 194, 2021.

[29] A. Gill, R. Garza, Y. Garcia-Mesa et al., "Heme oxygenase-1 promoter $(\mathrm{GT})(\mathrm{n})$ polymorphism predicts risk for neurocognitive impairment in HIV-infected individuals with higherrisk genotypes in African-Americans," Annals of Neurology, vol. 86, 2019.

[30] A. Gill, R. Garza, B. Gelman, S. Letendre, and D. Kolson, "Heme oxygenase-1 polymorphism associates with neuroimmune activation and neurocognitive impairment in HIVinfected individuals," Journal of Neurovirology, vol. 24, 2018.

[31] M. Vareille, F. Rannou, N. Thélier et al., "Heme oxygenase-1 is a critical regulator of nitric oxide production in enterohemorrhagic Escherichia coli-infected human enterocytes," Journal of Immunology, vol. 180, no. 8, pp. 5720-5726, 2008.

[32] A. T. Moussa, B. Singh, and A. N. Al-Dissi, "Immunohistochemical expression of nuclear factor erythroid-2-related factor 2 and heme oxygenase 1 in normal bovine lung and bovine lung infected with Mannheimia haemolytica," Canadian Journal of Veterinary Research-Revue Canadienne De Recherche Veterinaire, vol. 79, no. 2, pp. 81-86, 2015.

[33] D. Cattaneo, G. Orlando, V. Cozzi et al., "Linezolid plasma concentrations and occurrence of drug-related haematological toxicity in patients with gram-positive infections," International Journal of Antimicrobial Agents, vol. 41, no. 6, pp. 586-589, 2013.

[34] Y. Cai, D. Chai, M. E. Falagas et al., "Immediate hematological toxicity of linezolid in healthy volunteers with different body weight: a phase I clinical trial," Journal of Antibiotics, vol. 65, no. 4, pp. 175-178, 2012.

[35] L. Dou, D. Meng, Y. Dong et al., "Dosage regimen and toxicity risk assessment of linezolid in sepsis patients," International Journal of Infectious Diseases, vol. 96, pp. 105-111, 2020. 